\title{
The Role of Dietary Protein Intake and Resistance Training on Myosin Heavy Chain Expression
}

\author{
Colin D. Wilborn ${ }^{1}$ and Darryn S. Willoughby, FISSN ${ }^{1}$ \\ Exercise and Biochemical Nutrition Laboratory, Baylor University, Waco, TX. Journal of the \\ International Society of Sports Nutrition. 1(2):27-34, 2004. Address correspondence to \\ Colin_Wilborn@baylor.edu.
}

Received November 15, 2004/Accepted December 20, 2004/Published (online)

\begin{abstract}
During resistance training the muscle undergoes many changes. Possibly the most profound and significant changes are those that occur in the muscles contractile proteins. Increases in these contractile proteins are one of the primary factors contributing to myofibrillar hypertrophy. The most abundant muscle protein is myosin, which comprises $25 \%$ of the total muscle protein. Due to the large amount of skeletal muscle that is composed of myosin, changes in this fiber may have profound effects on skeletal muscle size and strength. The myosin molecule is made up of 6 subunits, 2 very large heavy chains, and 4 smaller light chains. The myosin heavy chain (MHC) accounts for $25-30 \%$ of all muscle proteins making its size an important factor in skeletal muscle growth. In conjunction with resistance training, dietary protein intake must be adequate to illicit positive adaptations. Although many studies have evaluated the role of dietary protein intake on skeletal muscle changes, few have evaluated the MHC specifically. Research has clearly defined the need for dietary protein and resistance training to facilitate positive changes in skeletal muscle. The purpose of this review was to evaluate the current literature on the effects of dietary protein and resistance training on the expression of the myosin heavy chain. Journal of the International
\end{abstract} Society of Sports Nutrition. 1(2):27-34, 2004

Key Words: Muscle Fiber, Contractile Protein, Protein Synthesis

\section{INTRODUCTION}

A combination of resistance training and proper diet elicits several physiological responses that enhance the structure and function of skeletal muscle. Research has clearly defined the benefits of resistance training (i.e., increased muscular endurance, strength, power, increased metabolism, decreases in body fat, etc) ${ }^{1-3}$. However one of the most profound changes seen is muscular hypertrophy, or more specifically myofibrillar and sarcoplasmic hypertrophy. Sarcoplasmic hypertrophy involves the growth of the sarcoplasm and non-contractile proteins ( $\alpha$-actinin, desmin, dystrophin, myomesins, nebulin, titin, and vinculin) that do not directly contribute to muscular force production $^{4,5}$. Filament area density decreases while cross-sectional area increases, without a significant increase in strength. Myofibrillar hypertrophy occurs due to increases in the number of myosin/actin filaments inside each sarcomere. This leads to increased strength and size of the contractile unit (actin, myosin, troponin, and tropomyosin) of muscle. The focus of this review will be the contractile components. These contractile components within each muscle fiber make up approximately $80 \%$ of the fiber. Thus, minimal increases in the number of contractile proteins may significantly increase the size of a fiber. In normal humans, skeletal muscle has been 
estimated to account for $40-50 \%$ of total body weight ${ }^{6}$, with as much as $50 \%$ of the total body protein being housed in the skeletal muscle ${ }^{6}$. The most abundant muscle protein is myosin comprising $25 \%$ of the total muscle protein $^{7}$. The myosin molecule is made up of 6 subunits, 2 very large heavy chains, and 4 smaller light chains. In a given muscle fiber the 2 large subunits are identical, although there are different heavy chain isoforms in different types of muscle fibers. Due to the large amount of skeletal muscle that is composed of myosin, changes in this fiber may have profound effects on skeletal muscle size and strength. The purpose of this review is to evaluate the current literature on the effects of dietary protein and resistance training on the expression of the myosin heavy chain (MHC). Although the focus of this review is the effects of dietary protein and resistance training on MHC changes, it is necessary to establish the foundation of the particular role that resistance training and protein intake play on skeletal protein balance.

\section{PROTEIN TURNOVER}

Skeletal muscle growth is possible only when muscle protein synthesis exceeds muscle protein breakdown. The body is in a continuous state of protein turnover. As old proteins are destroyed or degraded new ones are being synthesized. Thus, when synthesis of contractile proteins is occurring at a faster rate than degradation the net result is a positive protein balance or more specifically myofibrillar hypertrophy. Biolo and colleagues ${ }^{8}$ studied the effects of protein turnover and amino acid transport after resistance training in healthy untrained individuals. They found that after a bout of resistance training rates of protein turnover and amino acid transport were increased. However protein degradation was also increased above baseline values. Even though the net protein balance was increased over baseline, balance remained tilted in the favor of degradation. Protein synthesis increased by approximately $100 \%$ over baseline levels, where degradation increased 50\% over baseline. In another resistance training study Phillips and colleagues ${ }^{9}$ found similar findings to the Biolo study. The fractional synthesis rate (FSR) post exercise had increased significantly over baseline. However fractional breakdown rate was also increased. The results indicated a decrease in protein turnover, but still a negative turnover balance. There also appears to be a training effect on protein balance. After eight weeks of resistance training the acute exercise induced increases in muscle protein synthesis are reduced ${ }^{10}$. These findings concur with that of chronically resistance trained individuals ${ }^{9}$. In a $1999{ }^{9}$ study, the response of untrained subjects was compared to that of subjects with at least five years of resistance training. As previously noted, the results indicated that resistance trained individuals had a depressed protein turnover rate posttraining when compared to the untrained. However, eight weeks of resistance training resulted in chronic resting muscle protein synthesis (meaning a net positive muscle protein status was achieved).

Several studies have also investigated the duration of the elevated protein synthesis as a result of resistance training. Chesley and colleagues ${ }^{11}$ investigated the magnitude and time course for changes in muscle protein synthesis after a single bout of resistance exercise. Their results indicated that a single bout of heavy resistance exercise can increase biceps muscle protein synthesis for up to $24 \mathrm{~h}$ post exercise. A study done by MacDougall and colleagues ${ }^{12}$ examined the time course for elevated muscle protein synthesis by examining its rate at $36 \mathrm{hrs}$ following a training session. At $36 \mathrm{hrs}$ post exercise, muscle protein synthesis in the exercised muscle had returned to within $14 \%$ of the control. One conclusion from these studies is that following a bout of heavy resistance training, muscle protein synthesis increases rapidly and stays elevated for $24 \mathrm{hrs}$, and then declines rapidly so that at $36 \mathrm{hrs}$ it is not significantly different from baseline. Thus, these studies demonstrate the effectiveness of 
inducing changes in protein synthesis with resistance training. In fact, Ferrando et al ${ }^{13}$ found that moderate-resistance exercise can counteract the decrease in muscle protein synthesis seen during bed rest. Ferrando investigated two groups of healthy volunteers during 14 days of simulated bed rest. One group adhered to strict bed rest, and the second group engaged in leg resistance exercise every other day throughout bed rest. After 14 days of bed rest, muscle protein synthesis in the bed rest plus exercise group did not change and was significantly greater than in the bed rest group. These studies reflect the turnover of total muscle protein. Thus we can only speculate to the actual percentage of that synthesis rate that is specific to muscle proteins such as MHC. That said, very few studies have actually evaluated the synthesis of MHC.

Collectively, these studies suggest that resistance training will increase protein synthesis and slow degradation; however depending on the nature of the exercise stimulus, protein turnover may remain negative. Thus it is possible that resistance training combined with nutritional intervention may enhance the effects on protein synthesis.

\section{PROTEIN INTAKE}

It is interesting to note that previous studies have concluded that acute protein synthesis following resistance training is similar in fed 10 and fasted ${ }^{8,9}$ subjects. Only the rate of turnover changes slightly, but still remains in a negative balance. However, studies have found that with dietary protein or amino acid supplementation, muscle protein synthesis rate is increased. Biolo et al ${ }^{14}$ evaluated the interactions between resistance training and amino acid supplementation and the corresponding effects on protein kinetics. Six untrained men acted as subjects in this study. Each participant was infused with a mixed (phenylalanine, leucine, lysine, alanine, glutamine) amino acid solution approximately $0.15 \mathrm{~g} \cdot \mathrm{kg}^{-1} \cdot \mathrm{h}^{-1}$ for $3 \mathrm{~h}$. Baseline and post resistance training $(5 \times 10$ sets leg press and
$4 \times 8$ sets of nautilus squat, leg curl, and leg extension) measures were taken. The results revealed increased protein synthesis and no change in protein degradation. As noted previously, these results differ from that of the models that did not use amino acid supplementation. In those studies, protein synthesis increased, but protein degradation did as well. The findings of the Biolo study might indicate that increased availability of amino acids to the muscle after a bout of resistance training might contribute to enhanced anabolism. However in the Biolo study amino acids were infused in the subjects, which is hardly a realistic option for day-to-day resistance training. Tipton and colleagues ${ }^{15}$ recently investigated the effects of orally administered amino acids $(40 \mathrm{~g} \mathrm{CHO}$ (Placebo solution), 40g mixed amino acids (EAA + NEAA) or 40g Essential Amino Acids (EAA only + Arginine)) on post exercise net protein synthesis. They also sought to determine whether there would be a difference in the anabolic effect of amino acid supplementation if they used a mixed amino acid source or essential amino acids alone. The findings of this study indicated that postexercise amino acid supplementation elicits a positive protein balance as compared to the negative balance seen with resistance training alone. In fact, the results of this study were very similar to the infusion model. The Tipton study also concluded that supplementation with the essential amino acids alone is equivalent to that of a mixed amino acid supplement. Although research has concluded that post exercise amino acid supplementation has positive effect on protein synthesis, amino acid supplementation is not always an option. Thus Esmark et al ${ }^{16}$ investigated the timing of protein intake after exercise on muscle hypertrophy and strength. This study used a milk and soy protein supplement (containing $10 \mathrm{~g}$ protein (from skimmed milk and soybean), $7 \mathrm{~g}$ carbohydrate and $3.3 \mathrm{~g}$ lipid) instead of an amino acid mixture. Although protein synthesis was not calculated in this study, measurements of hypertrophy were made. As previously noted, muscular hypertrophy is the result of net 
protein synthesis. The results of the Esmark study indicated that skeletal muscle hypertrophy was significantly increased after resistance training when a protein supplement was taken. The findings also suggested that when the supplement was taken immediately after the training versus two hours later the hypertrophic response was greater. In the most recent study by Tipton and collegues, ${ }^{17}$ they evaluated the effects of casein and whey protein ingestion on protein balance after resistance training. Twenty three subjects consumed one of three drinks 1 hour after a bout of leg extensions. Subjects consumed either placebo, $20 \mathrm{~g}$ of casein protein, or $20 \mathrm{~g}$ of whey protein. The results indicated that ingestion of whey or casein protein after a bout of resistance exercise increases net muscle protein synthesis. In a recent review Rennie and collegues ${ }^{18}$ concluded that there is no doubt that increasing amino acid concentrations by intravenous infusion, meal feeding, or ingestion of free amino acids increases muscle protein synthesis. They also concluded that in the post exercise period increased availability of amino acids enhances muscle protein synthesis. These studies have clearly defined three things. First, resistance training decreases the net negative protein balance. By increasing protein synthesis at a greater rate than protein degradation, resistance training decreases net negative protein balance. Second, these findings suggest that resistance training decreases net negative protein balance regardless of fed or fasting state. Finally, amino acid or protein supplementation enhances protein synthesis and suppresses degradation, resulting in net protein synthesis. Recent studies have highlighted the importance of consuming different nutrients in varying quantities before or after resistance training. While a detailed breakdown of these considerations are beyond the scope of this review, the interested reader is encouraged to read the following articles that focus specifically on these considerations $19-23$

\section{MYOSIN HEAVY CHAIN ISOFORMS}

This review has established the role of resistance training and protein intake on muscle protein synthesis. However, the studies previously reviewed investigated net protein turnover and not MHC specifically. Adult skeletal muscle MHC is composed of many isoforms. There are at least three nonmuscle MHC isoforms, two smooth muscle isoforms, and at least eight skeletal muscle isoforms. The focus of this review will be on the adult isoforms: 1.) alpha cardiac beta or slow type I (as expressed in skeletal muscle); 2.) fast type Iia; 3.) fast type IIx/Iid; and 4.) fast type $I \mathrm{Ib}^{24}$. The majority of slow muscles (such as the soleus and vastus intermedius) express the slow type I MHC isoform with a proportion of type IIa. Type IIa is the slowest of the fast MHCs. In humans the mix of type I and IIa is almost equal in the soleus ${ }^{25}$. Mammals express two different fast MHC isoforms, type IIx and IIb. These isoforms are expressed in the small fast muscles such as the gastrocnemius-plantaris complex, the vastus, the extensor digitorum longus (EDL), and the tibialis anterior. The proportion of the isoform type varies from muscle to muscle and animal to animal ${ }^{25}$. However, humans do not express the type IIb isoform. Thus, in the human model fast muscle such as the vastus lateralis, EDL, and tibialis anterior type I, type IIa, and type IIx are expressed ${ }^{25}$. Protein expression of MHC genes is highly plastic and can be modulated by mechanical activity/inactivity factors. Changes in isoform composition are common as a result of exercise training. In fact, many hybrid patterns of MHC have been seen as a result of training ${ }^{24}$. A moderately active individual might have a MHC profile of the quadriceps (vastus) muscle that is $\sim 50 \%$ slow type I, $40 \%$ type IIa, and 10\% type IIx. Where a worldclass marathon runners and ultra-endurance athletes might have as much as $95 \%$ type I. Muscles of sprinters and powerlifters predominantly consist of the IIa/IIx MHC ${ }^{25}$. 


\section{MYOSIN HEAVY CHAIN SYNTHESIS}

As discussed previously, protein turnover is the rate at which protein is synthesized minus the rate at which it is degraded. Although this formula is quite simple, the process is complicated, and the protein turnover can occur on many levels. Protein turnover can be evaluated as whole-body (as discussed previously), as mixed muscle proteins, or as individual proteins (such as actin or myosin). Although the effects of mixed muscle protein synthesis give us information about exercise programs or dietary intervention, it does not speak to the specificity of the protein changes. By determining the synthesis rate of individual proteins we may gain a greater understanding of the adaptive response of exercise, bed rest, dietary intervention, and muscle wasting that occurs in a microgravity environment. A landmark study done on the synthetic rate of individual muscle proteins in humans was completed in $1997^{7}$. This study found that although the MHC composes at least $25 \%$ of muscle protein content, it contributed only $18 \%$ to the mixed muscle synthesis rate. Thus the synthesis rate of MHC is slower than that of the mixed muscle proteins. However Hasten et $\mathrm{al}^{26}$ found a direct correlation between mixed muscle and MHC protein synthesis rates after exercise, reporting that $\mathrm{MHC}$ synthesis rate accounted for $80 \%$ of the mixed muscle synthesis rate. These findings illustrate the profound effect resistance training has on the synthesis of MHC.

The role of dietary protein intake on protein turnover has been well established. As discussed previously the adequate requirement of essential amino acids is of paramount importance to reaching a net positive protein turnover. It is also clear that the ramification of restricted dietary protein intake is muscle wasting ${ }^{27}$. Recently a study ${ }^{28}$ found that consumption of an isoenergetic diet at the mean adult minimum protein requirement for 4 wk produced an $81 \%$ lower fractional synthesis rate of myosin heavy chain (MHC) proteins in vastus lateralis muscle than did consumption of an ample protein diet . They also found that protein deprivation altered the skeletal muscle myosin composition such that the proportion of the total myosin content represented by fasttwitch MHC IIx was 51\% lower than with ample intake. The most interesting finding was that the steady state content of MHC IIx messenger RNA did not differ in subjects consuming the minimum requirement of protein. This would suggest that the reduced proportion of MHC IIx arises from posttranscriptional events. They also found that a marker of protein degradation (3methylhistidine) was lower in the protein restricted group, suggesting that it is possible that both synthesis and degradation of MHC are slowed on a low protein diet.

\section{MYOSIN HEAVY CHAIN AND RESISTANCE TRAINING}

Studies clearly show that resistance-training induces adaptations in skeletal MHC isoform expression. These shifts predominantly involve rearrangement in the pattern of expression involving the fast MHC. Hather et $\mathrm{al}^{29}$ investigated the influence of resistance training on muscle fiber composition. The results show that after 19 weeks of heavy resistance training, IIb fiber composition decreased significantly, where, type IIa fiber increased. In a follow up study the authors ${ }^{30}$ of the same study used the samples to investigate the changes in the fast twitch fiber subtypes. The results show that after training, IIb MHC composition decreased and IIa MHC, in contrast, increased. These responses were essentially mirrored by alterations in fiber type distribution. The percentage of type IIb fibers decreased whereas the percentage of type IIa fibers increased. The control group showed no changes in $\mathrm{MHC}$ composition or fiber type distribution. These results suggest that heavy resistance training alters MHC composition in human skeletal muscle. Although it is not the focus of this review it is important to note that similar adaptations to resistance training are seen at the mRNA level as well as the protein level. 
Willoughby and Pelsue ${ }^{31}$ found that after 8 weeks of heavy resistance training MHC I, and IIa mRNA was significantly greater than the control group. However the type IIx mRNA significantly decreased after training. These findings agree with the findings of the previous studies that show a shift in the protein expression of MHC isoforms. Another study analyzed the distribution of MHC isoforms, fiber type composition, and fiber size of the vastus lateralis in a group of adult sedentary men before and after 3 months of heavy-load resistance training and, subsequently, after 3 months of detraining. Following the period of resistance training, MHC IIx content decreased significantly, with a corresponding significant increase in MHC IIa. After 3 months of detraining the amount of MHC IIX reached values that were higher than before and after resistance training. The results of this study indicated that there was a shift from type IIx fiber to type IIa after 12 weeks of resistance training 32. The findings of Andersen ${ }^{33}$ mirrored the results of the two previous studies, only they used sprinters as the subject group. These results suggest that heavy resistance training alters MHC composition in human skeletal muscle. Jurimae et al ${ }^{34}$ investigated whether 12 weeks of resistance training, which increased arm girth $(5 \%)$ and forearm extensor strength (39\%), also altered the MHC characteristics of the triceps brachii muscle. The results indicated that resistance training changed the contractile protein profile of trained skeletal muscle. However, changes in MHC isoform composition in the first 12 weeks of training were not implicated in the development of 1RM triceps pushdown strength. The results of this study might infer that changes in MHC composition contribute to increases myofibrillar hypertrophy. For this to be true, resistance training could not only change the composition of the MHC, but it would have to increase the synthesis of the MHC. Welle and collegues ${ }^{35}$ investigated the synthesis of myofibrillar proteins in young and older adults after three months of resistance training. The results indicated that resistance training significantly increased lean body mass. However this study did not find any significant changes in myofibrillar protein synthesis. This study is in contrast to others. Chesley ${ }^{11}$ found that muscle protein synthesis was increased significantly after resistance training as did Hasten et $\mathrm{al}^{26}$. Hasten investigated the changes in mixed muscle protein, myosin heavy chain, and actin proteins before and after two weeks of resistance training. They evaluated seven subjects with a mean age of 27 and seven subjects with a mean age of 80 years old. In the young subjects the MHC synthesis rate increased $88 \%$ and the mixed muscle protein increased $121 \%$. In the older group, MHC synthesis rates increased $105 \%$ and mixed muscle protein synthesis increased $182 \%$ over baseline. Thus MHC synthesis accounted for $80 \%$ and $65 \%$ respectively in the younger and older individuals total mixed muscle protein synthesis. Balagopal et $\mathrm{al}^{5}$ also found a significant training effect on MHC synthesis rate. After three months of resistance training the fractional synthesis rate of $\mathrm{MHC}$ increased $47 \%$ and mixed muscle protein synthesis increased $56 \%$. It is clear that much of the current literature agrees that resistance training increases the synthesis of myofibrillar proteins and that $\mathrm{MHC}$ accounts for a large portion of that synthesis rate.

\section{CONCLUSION}

The purpose of this review was to evaluate the current literature on the role and importance of dietary protein and resistance training on the expression of the skeletal muscle isoforms of the myosin heavy chain. The importance of the MHC can almost be quantified by its sheer size, accounting for 25 $-30 \%$ of all muscle proteins. However, its plasticity also makes up the muscle fibers diversity. Essentially, the MHC dictates how the muscle will react to an induced load, such as that of exercise. The adaptability of the MHC allows for the muscle to conform (to some degree) to the given stress placed on the muscle. The research has clearly defined that resistance training increases the synthesis rate as well as impacting the specific isoform 
expression of the MHC gene. Although further research needs to be done, past research has also determined that adequate protein intake is a necessity for MHC isoform expression. The role of specific amino acids and dietary protein on protein turnover is well established, however there needs to be further investigation into the role of dietary intervention on the MHC family. Future studies may investigate the role of various dietary supplements as well as various resistance training paradigms on expression of the myosin heavy chain family.

\section{REFERENCES}

1. Booth, FW and DB Thomason: Molecular and cellular adaptation of muscle in response to exercise: perspectives of various models. Physiol Rev 1991, 71:541-85.

2. McCall, GE, WC Byrnes, SJ Fleck, et al.: Acute and chronic hormonal responses to resistance training designed to promote muscle hypertrophy. Can J Appl Physiol 1999, 24:96-107.

3. Deschenes, MR and WJ Kraemer: Performance and physiologic adaptations to resistance training. Am J Phys Med Rehabil 2002, 81:S3-16.

4. Nikituk B, SN: adaptive mechanisms of muscle fibers to exercise and possibilities for controlling them. Teoriya i Praktika Fizischeskoi Kultury 1990, 5:11-14.

5. Siff MC., VY, Supertraining: A Textbook on the Biomechanics and Physiology of Strength Conditioning for all Sport. 1996, Pittsburgh: Sports Support Syndicate.

6. Houston, ME: Gaining weight: the scientific basis of increasing skeletal muscle mass. Can J Appl Physiol 1999, 24:305-16.

7. Balagopal, P, O Ljungqvist, and KS Nair: Skeletal muscle myosin heavy-chain synthesis rate in healthy humans. Am J Physiol 1997, 272:E45-50.

8. Biolo, G, SP Maggi, BD Williams, et al.: Increased rates of muscle protein turnover and amino acid transport after resistance exercise in humans. Am J Physiol 1995, 268:E514-20.

9. Phillips, SM, KD Tipton, AA Ferrando, et al.: Resistance training reduces the acute exercise-induced increase in muscle protein turnover. Am J Physiol 1999, 276:E118-24.

10. Phillips, SM, G Parise, BD Roy, et al.: Resistance-training-induced adaptations in skeletal muscle protein turnover in the fed state. Can J Physiol Pharmacol 2002, 80:1045-53.

11. Chesley, A, JD MacDougall, MA Tarnopolsky, et al.: Changes in human muscle protein synthesis after resistance exercise. J Appl Physiol 1992, 73:1383-8.

12. MacDougall, JD, MJ Gibala, MA Tarnopolsky, et al.: The time course for elevated muscle protein synthesis following heavy resistance exercise. Can J Appl Physiol 1995, 20:480-6.

13. Ferrando, AA, KD Tipton, MM Bamman, et al.: Resistance exercise maintains skeletal muscle protein synthesis during bed rest. J Appl Physiol 1997, 82:807-10.

14. Biolo, G, KD Tipton, S Klein, et al.: An abundant supply of amino acids enhances the metabolic effect of exercise on muscle protein. Am J Physiol 1997, 273:E122-9.

15. Tipton, KD, AA Ferrando, SM Phillips, et al.: Postexercise net protein synthesis in human muscle from orally administered amino acids. Am J Physiol 1999, 276:E628-34.

16. Esmarck, B, JL Andersen, S Olsen, et al.: Timing of postexercise protein intake is important for muscle hypertrophy with resistance training in elderly humans. J Physiol 2001, 535:301-11.

17. Tipton, KD, TA Elliott, MG Cree, et al.: Ingestion of casein and whey proteins results in muscle anabolism after resistance exercise. Med Sci Sports Exerc 2004, 36:2073-2081.

18. Rennie, MJ, H Wackerhage, EE Spangenburg, et al.: Control of the size of the human muscle mass. Annu Rev Physiol 2004, 66:799-828.

19. Tipton, KD and RR Wolfe: Protein and amino acids for athletes. J Sports Sci 2004, 22:65-79.

20. Wolfe, RR: Regulation of muscle protein by amino acids. J Nutr 2002, 132:3219S-3224.

21. Tipton, KD, E Borsheim, SE Wolf, et al.: Acute response of net muscle protein balance reflects 24-h balance after exercise and amino acid ingestion. Am J Physiol Endocrinol Metab 2003, 284:E76-E89. Rasmussen, BB and SM Phillips: Contractile and nutritional regulation of human muscle growth. Exerc Sport Sci Rev 2003, 31:127-131.

23. Borsheim, E, A Aarsland, and RR Wolfe: Effect of an amino acid, protein, and carbohydrate mixture on net muscle protein balance after resistance exercise. International Journal of Sport Nutrition and Exercise Metabolism 2004, 14:255-271. Weiss, A and LA Leinwand: The mammalian myosin heavy chain gene family. Annu Rev Cell Dev Biol 1996, 12:417-39. Baldwin, KM and F Haddad: Effects of different activity and inactivity paradigms on myosin heavy chain gene expression in striated muscle. J Appl Physiol 2001, 90:345-57.

26. Hasten, DL, J Pak-Loduca, KA Obert, et al.: Resistance exercise acutely increases MHC and mixed muscle protein synthesis rates in 78-84 and 23-32 yr olds. Am J Physiol Endocrinol Metab 2000, 278:E620-6.

27. Castaneda, C: Muscle wasting and protein metabolism. J. Anim. Sci. 2002, 80:E98-E105.

28. Brodsky IG, SD, Hornberger TA, Goldspink P, Yarasheski KE, Smith S, Kukowski J, Esser K, Bedno S.: Isoenergetic dietary protein restriction decreases myosin heavy chain IIx fraction and myosin heavy chain production in humans. J. Nutr. 2004, 134:328-334.

29. Hather, BM, PA Tesch, P Buchanan, et al.: Influence of eccentric actions on skeletal muscle adaptations to resistance training. Acta Physiol Scand 1991, 143:177-85. 
30. Adams, GR, BM Hather, KM Baldwin, et al.: Skeletal muscle myosin heavy chain composition and resistance training. J Appl Physiol 1993, 74:911-5.

31. Willoughby, DS and S Pelsue: Effects of High-Intensity Strength Training on Steady State Myosin Heavy Chain Isoform Expression. Hournal of Exercise Physiology online 2000, 3:

32. Andersen, JL and P Aagaard: Myosin heavy chain IIX overshoot in human skeletal muscle. Muscle Nerve 2000, 23:1095104.

33. Andersen, JL, H Klitgaard, J Bangsbo, et al.: Myosin heavy chain isoforms in single fibres from $\mathrm{m}$. vastus lateralis of soccer players: effects of strength-training. Acta Physiol Scand 1994, 150:21-6.

34. Jurimae, J, PJ Abernethy, K Blake, et al.: Changes in the myosin heavy chain isoform profile of the triceps brachii muscle following 12 weeks of resistance training. Eur J Appl Physiol Occup Physiol 1996, 74:287-92.

35. Welle, S, C Thornton, and M Statt: Myofibrillar protein synthesis in young and old human subjects after three months of resistance training. Am J Physiol 1995, 268:E422-7. 